\title{
Teledermatology management of difficult-to-treat dermatoses in the Faroe Islands
}

\author{
Rune Kjærsgaard Andersen ${ }^{1,2} \bowtie$, Gregor Borut Ernst Jemec ${ }^{1,2}$
}

\begin{abstract}
Introduction: Teledermatology is a useful tool for underserved areas. However, because it is not without costs, knowledge of the burdens incurred by teledermatology treatment would be valuable for future types of management.

Methods: All specialist dermatology services in the Faroe Islands have been provided by our department through a mix of standard and teledermatology consultations. We performed a retrospective review of the teledermatology database from its inauguration in 2003 to November 2018. The ratio of consultations per patient (CPP) was calculated as a way to investigate any disproportionate teledermatology burden per patient with a specific disease.

Results: The most common diagnoses were psoriasis, dermatitis, atopic dermatitis, and acne. Among the most frequent CPP diagnoses were bullous pemphigoid, hidradenitis suppurativa, lichen sclerosus, and granuloma annulare.

Conclusions: Dermatological conditions that require paraclinical assistance for diagnosis, hands-on examination, or treatment with either biologics or surgery will all result in high CPP. However, teledermatology still has a place in the treatment of high-CPP dermatoses in rural areas because there is no other alternative. For non-rural areas, CPP can be used as a way to identify those dermatoses that are not advantageous to treat using telemedicine, but instead best benefit from standard face-to-face consultation.
\end{abstract}

Keywords: teledermatology, dermatosis treatment, teledermatology consultation, difficult to treat

Received: 8 April 2019 | Returned for modification: 29 May 2019| Accepted: 3 June 2019

\section{Introduction}

Telemedicine is particularly useful in fields that rely strongly on visual assessment of morphological appearance, such as dermatology (1). It can bring expert dermatological assessment of cases to underserved areas like rural Australia (2), Sub-Saharan Africa (3), and the Faroe Islands (4). From the perspective of a specialist, teledermatology is frequently proposed as a means of reducing not only the burden of treatment but also cost $(1,5)$. However, teledermatology is not without costs in itself. We therefore identified diseases with a high number of consultations as a possible proxy for disease severity or complexity. When we noticed that knowledge regarding the relative burden of teledermatology treatment of difficult dermatoses was lacking, we decided to investigate the teledermatology challenge of skin diseases because increased knowledge of their places in teledermatology may help shape future decisions regarding this type of management.

\section{Methods}

\section{The Faroe Islands}

The Faroe Islands are a group of 18 islands in the north Atlantic, located between the UK, Iceland, and Norway. The islands cover $1,396 \mathrm{~km}^{2}$ and have a population of 49,864 (in 2017). Specialist dermatology service is exclusively provided by Zealand University Hospital in Denmark (approximately 1,500 km away).

\section{Health services provided}

All specialist dermatology services in the Faroe Islands are provided by the Department of Dermatology at Zealand University
Hospital through a mix of standard consultations (80o/year) and store-and-forward teledermatology consultations (approximately $2,400 /$ year). Patients are referred to the dermatology service by general practitioners, and they are triaged to either standard physical consultations or telemedicine, depending on the information provided. Patients seen in standard face-to-face consultations are subsequently transferred to telemedicine as soon as clinically possible. Telemedicine consultations therefore represent both standard follow-up as well as emergency assessment of flares because the telemedical capacity is much greater than that of face-to-face consultations.

\section{Analysis}

We performed a retrospective review of the teledermatology database from its inauguration in 2003 to November 2018. The 100 most frequent diagnoses were selected (the number 100 was arbitrarily chosen) to avoid rare diseases with only a single diagnosed patient seen multiple times. All diseases were then pooled into groups where applicable (i.e., psoriasis and psoriasis vulgaris were grouped under "psoriasis"), and descriptive statistics and simple comparisons between diagnostic entities were calculated. We calculated the ratio of consultations per patient (CPP) as a way to investigate any disproportionate teledermatology burden for each patient with a specific disease.

\section{Results}

From 2003 to 2018, 10,713 Faroese were diagnosed by a dermatologist and managed by teledermatology across 803 different diagnoses. Descriptive statistics of the 10 most common diagnoses are shown in Table 1. The most common dermatoses were psoriasis 
(1,081 patients), dermatitis (864 patients), atopic dermatitis (816 patients), and acne (601 patients). The top 10 most common diagnoses covered $44.4 \%$ of all teledermatology patients and $49.4 \%$ of all teledermatology consultations. Table 2 shows the 10 diseases with the highest teledermatology burden/CPP. These are bullous pemphigoid (CPP 1.67), hidradenitis suppurativa (CPP 1.43), lichen sclerosus (CPP 1.36), psoriasis (CPP 1.27), and granuloma annulare (CPP 1.23).

\section{Discussion}

Significant differences were found in the CPP for different diagnoses. Among the resource-heavy diagnoses, we find hidradenitis suppurativa, psoriasis, atopic dermatitis, hand eczema, bullous pemphigoid, lichen sclerosus, granuloma annulare, and balanitis. Different mechanisms may be suspected. Bullous pemphigoid, hidradenitis suppurativa, and lichen sclerosus are generally recognized as difficult-to-treat dermatoses. Bullous pemphigoid requires histopathology and immunofluorescence for diagnosis and needs to be followed up for prolonged periods of time (6). Hidradenitis suppurativa used to be an orphan disease, where the only treatment for severe cases is surgery and tumor necrosis factor (TNF) antibodies (7). Lichen sclerosus is a chronic inflammatory disease for which the diagnosis is clinical but patients may require a biopsy to rule out vulvar intraepithelial neoplasia. The disease may further require surgery to alleviate synechiae complications (8). In contrast, granuloma annulare is a benign self-limiting inflammatory condition without a gold standard for treatment but with a wide array of therapeutic options ranging from photo- therapy and injections to cryosurgery (9). Balanitis is a common disorder with multiple infectious and non-infectious etiologies. The disease increases the risk of penile cancer and the development of ulcerations and phimosis. The diagnosis is clinical but often requires swabs and cultures to rule out sexually transmitted disease. The treatment is simple, but circumcision can become relevant (10). It is speculated that patients' coping and demands for treatment contribute to the high CPP for granuloma annulare. Similar arguments are suggested for balanitis. Thus, a range of diagnosis-specific factors may be hypothesized: treatment-associated factors, factors associated with diagnostic testing, or psychosocial patient-related factors may influence the CPP.

A previous study in rural Australia found that dermatologists requested biopsy for further information in $29.2 \%$ of the cases referred for teledermatology (2). A frequently promoted point of teledermatology is that it is supposed to be quick and easy for the dermatologist, offering a means of reducing both the burden of treatment and cost. Thus, teledermatology should be used for simple diseases, for which patients do not require multiple consultations. Physical absence of a dermatologist impose restrictions on both examination and treatment. As is apparent from our data, many difficult dermatoses require a physical dermatologic presence. This is because proper diagnosis may require help from other specialties (e.g., for bullous pemphigoid); because regular disease activity is troublesome, as in the case of sinus tracts (e.g., for hidradenitis suppurativa) or flares (e.g., for atopic dermatitis); or due to supervision to adherence and monitoring for adverse effects of systemic treatment (e.g., for psoriasis and acne). These restrictions can explain at least some of the disproportionate bur-

Table 1 | The ten most frequent diagnoses as measured by the number of patients listed under that diagnosis.

\begin{tabular}{|c|c|c|c|c|c|c|}
\hline $\begin{array}{l}\text { Rank among } 803 \\
\text { diagnoses }\end{array}$ & ICD10 codes & Diagnosis & $\begin{array}{c}\text { Patients, } \\
n \text { (\% of total) }\end{array}$ & $\begin{array}{c}\text { Teledermatology } \\
\text { consultations (\% of total) }\end{array}$ & Average age & $\mathrm{CPP}$ \\
\hline 1 & $\begin{array}{c}\text { L40.9, L40., L40.4, } \\
\text { L40.3, L40.0C, L40.8A }\end{array}$ & Psoriasis & $1,081(10.1)$ & $1,372(11.5)$ & 51.2 & 1.27 \\
\hline 2 & $\begin{array}{c}\mathrm{L} 30.9, \mathrm{~L} 30.0, \mathrm{~L} 30.8 \mathrm{C} \\
\text { L30., L30.8A }\end{array}$ & Dermatitis & $864(8,1)$ & 939 (7.9) & 53.3 & 1.09 \\
\hline 3 & $\begin{array}{c}\mathrm{L} 20.9, \mathrm{~L} 20.8 \mathrm{~A}, \mathrm{~L} 20.0 \\
\mathrm{~L} 20.8 \mathrm{~B}, \mathrm{~L} 20.8\end{array}$ & Atopic dermatitis & $816(7.6)$ & 964 (8.1) & 19.4 & 1.18 \\
\hline 4 & L70.9, L70.0, L70. & Acne & $601(5.6)$ & $731(6.1)$ & 29.5 & 1.22 \\
\hline 5 & D22.9, D22.5, D22.9E & Nevi (incl. dysplastic) & $440(4.1)$ & $470(4.0)$ & 34.7 & 1.07 \\
\hline 6 & L71.9, L71., L71.8 & Rosacea & $368(3.4)$ & $398(3.3)$ & 55.7 & 1.08 \\
\hline 7 & L21.9, L21.0, L21. & Seborrheic dermatitis & $273(2.5)$ & $295(2.5)$ & 49.3 & 1.08 \\
\hline 8 & $\mathrm{~L} 30.8 \mathrm{H}$ & Hand eczema & $253(2.4)$ & $301(2.5)$ & 44.8 & 1.19 \\
\hline 9 & L23.9, L23. & $\begin{array}{l}\text { Allergic contact } \\
\text { dermatitis }\end{array}$ & $198(1.8)$ & 209 (1.8) & 53.3 & 1.06 \\
\hline 10 & L50.9, L50.8A, L50.8D & Urticarial & $191(1.8)$ & $207(1.7)$ & 41.8 & 1.08 \\
\hline Total & & & 10,713 & 11,893 & 47 & 1.07 \\
\hline
\end{tabular}

CPP $=$ ratio of consultations per patient. ICD10 codes from https://icd.who.int/browse10/2016/en.

Table 2 | Ten diagnosis with the highest teledermatology burden per patient.

\begin{tabular}{|c|c|c|c|c|c|c|}
\hline $\begin{array}{l}\text { Frequency among } \\
803 \text { diagnoses }\end{array}$ & ICD10 codes & Diagnosis & Patients, $n$ & $\begin{array}{c}\text { Teledermatology } \\
\text { consultations }\end{array}$ & Average age & CPP \\
\hline 100 & L 12.0 & Bullous pemphigoid & 18 & 30 & 88.1 & 1.67 \\
\hline 65 & L 73.2 & Hidradenitis suppurativa & 30 & 43 & 38.2 & 1.43 \\
\hline 62 & L 90.0 & Lichen sclerosus & 31 & 42 & 52.3 & 1.36 \\
\hline 1 & $\begin{array}{c}\text { L40.9, L40., L40.4, L40.3, } \\
\text { L40.0C, L40.8A }\end{array}$ & Psoriasis & 1,081 & 1,372 & 51.2 & 1.27 \\
\hline 52 & L 92.0 & Granuloma annulare & 35 & 43 & 41.6 & 1.23 \\
\hline 4 & L70.9, L70.0, L70. & Acne & 601 & 731 & 29.5 & 1.22 \\
\hline 57 & N48.1A & Balanitis & 33 & 40 & 60.4 & 1.21 \\
\hline 8 & L30.8H & Hand eczema & 253 & 301 & 44.8 & 1.19 \\
\hline 3 & $\begin{array}{l}\text { L20.9, L20.8A, L20.0, } \\
\text { L20.8B, L20.8 }\end{array}$ & Atopic dermatitis & 816 & 964 & 19.4 & 1.18 \\
\hline 53 & C43.9 & Malignant melanoma & 34 & 40 & 57.6 & 1.18 \\
\hline
\end{tabular}

$\mathrm{CPP}=$ ratio of consultations per patient. ICD10 codes from https://icd.who.int/browse10/2016/en. 
den of these diseases on the teledermatology service. Because teledermatology is used as an adjunct to standard face-to-face consultations, the transfer of all high-CPP consultations to standard consultations would place an even greater burden on these and may therefore not be feasible.

Limitations of this study are that the database only registered the number of teledermatology consultations, not the standard consultations. There is a possibility that, if it were quickly decided that a complicated case should be seen in a standard consultation, this would falsely lower the CPP. This may explain why none of the diagnoses presents with a CPP above two.

\section{Conclusions}

Difficult-to-treat dermatoses and dermatological conditions that

\section{References}

1. Lee KJ, Finnane A, Soyer HP. Recent trends in teledermatology and teledermoscopy. Dermatol Pract Concept. 2018;8:214-23.

2. Byrom L, Lucas L, Sheedy V, Madison K, Mclver L, Castrisos G, et al. Tele-Derm National: a decade of teledermatology in rural and remote Australia. Aust J Rural Health. 2016;24:193-9.

3. Tsang MW, Kovarik CL. The role of dermatopathology in conjunction with teledermatology in resource-limited settings: lessons from the African Teledermatology Project. Int J Dermatol. 2011;50:150-6.

4. Jemec GB, Heidenheim M, Dam TN, Vang E. Teledermatology on the Faroe Islands. Int J Dermatol. 2008;47:891-3.

5. Muir J. Telehealth: the specialist perspective. Aust Fam Physician. 2014;43:82830. require either paraclinical assistance for diagnosis, hands-on examination, or treatment that involves regular follow-up will all result in a higher teledermatology burden. Currently there is no other alternative for high-CPP diseases besides teledermatology, which therefore still has a place in the care of difficult-to-manage dermatoses in rural areas. The authors' suggestion is that for dermatologists invested in telemedicine in non-rural areas CPP can be used as a way to identify dermatoses that are not advantageous to treat via telemedicine. It is speculated that patients with a high-CPP disease are more appropriately seen face-to-face, in turn freeing up resources for teledermatology, which is then more cost-effectively spent for treating low-CPP dermatoses.
6. Bagci IS, Horvath ON, Ruzicka T, Sardy M. Bullous pemphigoid. Autoimmun Rev. 2017;16:445-55.

7. Vekic DA, Frew JW, Woods J, Cains GD. Adopting the orphan: the importance of recognising hidradenitis suppurativa as a systemic auto-inflammatory disease. Australas J Dermatol. 2016;57:69-70.

8. Perez-Lopez FR, Vieira-Baptista P. Lichen sclerosus in women: a review. Climacteric. 2017;20:339-47.

9. Wang J, Khachemoune A. Granuloma annulare: a focused review of therapeutic options. Am J Clin Dermatol. 2018;19:333-44.

10. Wray AA, Khetarpal S. Balanitis. StatPearls. Treasure Island (FL): StatPearls Publishing LLC; 2018. 